\title{
Does food intolerance have any role in the aetiology and management of rheumatoid disease?
}

\author{
L GAIL DARLINGTON \\ From the Epsom District Hospital, Epsom, Surrey
}

SUMmaRY Dietary therapy for rheumatoid disease has been used by patients for decades. Until recently, orthodox medical opinion has tended to ignore the subject, but interesting results from recent studies suggest that further investigation would now be appropriate. It is possible that food (which consists of many antigens regularly entering the body) could be responsible for altering immunological function. Be that as it may, it would seem logical, particularly in view of recent uncertainty about the efficacy and/or safety of various drugs used in the management of rheumatoid disease, to determine scientifically whether dietary manipulation has any value in the management of these rheumatoid patients.

The role of food in rheumatoid disease is a topic on which opinions have been held strongly but conflictingly and mainly in the context of a therapeutic rather than an aetiological role.

For those of us with an orthodox rheumatological training the subject of diet in rheumatoid disease has the disturbing flavour of fringe medicine and tends to be dismissed accordingly. Also, the placebo effect associated with any new therapy will often produce apparent benefit and, particularly in view of the relapses and remissions in the natural history of rheumatoid disease, a placebo effect must not be mistaken for improvement.

This discussion on the possible role of food and chemical intolerance in the aetiology and therapy of rheumatoid disease is approached, therefore, in the full knowledge of the pitfalls inherent in assessing the subject. It is also written, however, in the belief that the data summarised below are sufficiently interesting to warrant further, objective assessment.

Furthermore, in spite of immense effort made in research, there is still considerable uncertainty about the aetiology and management of rheumatoid disease and it would seem unreasonable to dismiss without investigation any new suggestions or ideas simply because the approach is novel and the

Accepted for publication 25 July 1985.

Correspondence to Dr L G Darlington, Epsom District Hospital, Dorking Road, Epsom, Surrey KT18 7EG. mechanism unknown. Not infrequently in the history of medicine explanations have followed clinical advances.

Most of the work on the role of food intolerance in the aetiology of rheumatoid disease has been published in journals read primarily by allergists, and between allergists and rheumatologists there has been little communication. ${ }^{1}$

Speculation about food intolerance in rheumatology dates from the early part of this century, and a variety of arthritides and myalgias have been attributed to food intolerance. ${ }^{2-5}$

In 1935 Gudzent $^{6}$ reported that arthritis could be exacerbated by the ingestion of certain foods and, in 1924 and again in 1944 , Turnbull ${ }^{78}$ reported relief from rheumatoid arthritis by food exclusions.

In 1949 Zeller $^{9}$ described improvement in patients with polyarthritis by diagnosis and treatment of food intolerance, and this work was later extended by Randolph. ${ }^{10}$

Well controlled clinical trials of dietary manipulation in rheumatoid disease are so far limited in number.

In a controlled study in 1979 Sköldstam, Larsson, and Lindström ${ }^{11}$ partially fasted 16 patients with classical rheumatoid arthritis for seven to 10 days and then gave them a lactovegetarian diet for a nine-week period. After fasting five out of 15 patients showed objective signs of improvement 
compared with only one of the controls. At the conclusion of the lactovegetarian diet, however, only one patient still showed objective improvement and there were no differences between diet patients and controls. These results would be consistent with initial improvement on removing foods during the fast, followed by deterioration in all but one patient with the reintroduction of a more normal diet. Nevertheless, the numbers were small and care must be taken in their interpretation.

In 1980 Hicklin et al. ${ }^{2}$ reported clinical improvement in 24 of 72 rheumatoid patients treated with exclusion dieting.

In 1981 Parke and Hughes ${ }^{13}$ reported a most interesting case of a rheumatoid patient who responded well to restriction of cheese and dairy products and, in the same year, Williams ${ }^{14}$ described dramatic improvement in a rheumatoid patient with intolerance of corn.

In 1982 Dr Mansfield and I completed a prospective pilot study designed to investigate, firstly, whether intolerant reactions to foods, inhalants, and environmental chemicals could be demonstrated among 21 patients with classical or definite rheumatoid disease and, secondly, whether withdrawal of allergenic substances resulted in objective reduction in rheumatoid activity.

This study showed significant benefit for the group treated by diet. It was, however, an uncontrolled study, designed simply to tell us whether there was any point in setting up a controlled study, and its results must be viewed with that knowledge. Our prospective, blind study controlled against placebo is now complete, having investigated the role of food intolerance in the management of rheumatoid disease and will soon be published. Again patients receiving dietary therapy improved significantly.

In 1983 Panush et al. ${ }^{15}$ in Florida undertook a 10 -week controlled double blind randomised trial in 26 patients to investigate the Dong diet for rheumatoid arthritis. This is a specific, popular diet free from additives, preservatives, fruit, red meat, herbs, and dairy products. Six patients on the Dong diet and five patients on the 'placebo' diet improved by objective criteria. Two patients on the experimental diet improved very much, elected to remain on the experimental diet after the study period, continued to improve, and noted exacerbations of disease after eating non-experimental diet food. Panush et al. stated that their study failed to provide evidence of overall clinical benefit for the diet but that the results were not inconsistent with the possibility that individualised dietary manipulations might be beneficial for selected patients with rheumatoid disease.

It is perhaps not surprising that this study of the
Dong diet did not yield dramatic improvemento since cereals such as wheat and corn were permitte on the diet and many arthritic patients investigate for food intolerance seem to have intolerance of these foods.

Be that as it may, Ziff ${ }^{16}$ reviewed Panush's pape and also current interesting evidence suggesting a effect of diet on autoimmune disease and conclude 4 that there appeared to be a case for very carefullo controlled investigation of diet in the treatment of rheumatoid arthritis, though such investigation could be fraught with difficulties.

In 1984 Kroker et al. ${ }^{17}$ published a multicentro study which included 43 patients with definite of classical rheumatoid arthritis who underwent is water fast lasting for one week under controlled environmental conditions. Seven parameters of arthritic activity all improved significantly during the fast $(\mathrm{p}<0.01)$.

In January 1985 Kremer et al. ${ }^{18}$ described thei? manipulation of dietary fatty acids in patients with rheumatoid arthritis. In this study patients were investigated in a 12-week, prospective, double blin $\mathbb{P}$ study. Seventeen patients took an experimental die high in polyunsaturated fats and low in saturate\& fats with a daily supplement of $1.8 \mathrm{~g}$ eicosace pentaenoic acid, while 20 patients took a contos diet with a placebo supplement. The resitis favoured the experimental group at 12 weeks fop morning stiffness and the number of tender joints After stopping the diet the experimental group deteriorated significantly in patients' and physicians。 evaluation of disease activity, pain assessment, an\& number of tender joints.

The coverage of Kremer's paper by the media led to the name 'The Eskimo Diet' being used to describe the reduction in dairy produce and animal. fat in this study and the use of a diet rich in fish an\& fish oil.

This summarises the reports that have appeared in. the medical literature on this subject.

The continued activity of rheumatoid disease often for many years, is consistent with the cono tinued presence of a stimulus to maintain it. Since many antigens gain entrance to the body via the intestinal tract through the consumption of food, if is possible that, at least in some patients, food coul $\Phi$ be the source of antigens which perpetuate the rheumatoid process.

It has also been suggested ${ }^{19}$ that rheumatoif arthritis is a relatively recent disease with no convincing evidence of its existence before the latte을 part of the 17 th century. If the disease really is o relatively recent origin, an environınental causè becomes a possibility and modern diets coulf perhaps be implicated. 
One theory put forward by clinical ecologists is that intolerant reactions to food are 'masked' to produce rheumatoid arthritis. A brief description of such masking may be helpful.

Some people are aware that they are intolerant of certain foods. These are generally foods which are eaten infrequently, such as sea food, and the problem is usually overcome simply by avoidance of the offending items.

Intolerance of commonly eaten foods is also described by allergists and is said to be less readily detected or avoided. The theory of masked food intolerance suggests that if, for example, one develops intolerance to milk early in life, one may at first have an acute reaction to milk but, in time, if the intolerance is not recognised and if milk continues to be consumed, the symptoms become more generalised, less easily detected, and one day's symptoms blur into those of the next day, i.e., they become masked.

How then should a patient be investigated for food intolerance? On the assumption that serious alternative disease has been excluded, various methods may be used: (a) dietary exclusion of allergenic foods, followed by deliberate reintroduction of foods in a certain sequence; $(b)$ radioallergosorbent test (RAST); (c) the intradermal provocation method.

\section{Dietary exclusion method}

The dietary exclusion method is delightful in its simplicity. If a patient with constant symptoms is fasted for six days on mineral water alone, his symptoms should clear completely if they are caused only by food intolerance-older patients may take seven or eight days to clear.

As outpatients, many people find a total fast unacceptable, so a useful hypoallergenic diet of mineral water, cod, and pears may be used: cod and pears represent two foods of which the patient is unlikely on a statistical basis to be intolerant. They are acceptable, nutritious, and available throughout the year. Complete compliance with the prescribed diet is emphasised, since this is crucial for accurate interpretation. The patient should stop smoking (since cigarettes may contain traces of sugar used in curing the tobacco) and no medication is allowed, since most tablets contain corn starch as a filler and corn is a common food of which many patients are intolerant.

After the six days of this elimination dieting foods are next reintroduced one at a time and in a certain order to see whether any symptoms are produced. If symptoms are produced, that item is then kept out of the diet; if they are not produced, then that food can be considered to be 'safe' and included from then on in the dietary programme.

Testing of approximately 70 commonly eaten food items takes about six weeks. At the end of this programme many patients will have found a number of foods which produced symptoms (and indeed many normal people on using such an elimination dieting regimen will develop symptoms). The question remains whether these symptoms are clinically relevant.

\section{The radioallergosorbent test (RAST)}

The radioallergosorbent test (RAST) measures allergen-specific IgE antibodies but does not provide any information about the allergenicity of a given food in a particular person at a given time. It is an adjunctive test to confirm clinical suspicions but has major limitations: the test is expensive, has a significant false positivity rate, and the number of foods which can be tested is limited.

\section{The intradermal provocation method for diagnosis and management}

The unsatisfactory results of conventional and intradermal skin tests have led to their virtual abandonment by many clinicians. The end-point dilution method of skin testing, however, was developed by Lee in 1959 and described in 1964 in conjunction with Rinkel et al. ${ }^{20}$ The value of the technique is still not proved, but its adherents describe it as an effective diagnostic procedure.

Many people believe that rheumatoid disease is a diagnosis applied to a non-homogeneous population of patients whose disease probably arises as a result of a variety of trigger factors. These may be hormonal, viral, or of unknown aetiology, and it would seem possible that diet could be one such trigger in some people.

There is even some fascinating, recent evidence on the subject of increased gut permeability in rheumatoid patients when treated with nonsteroidal anti-inflammatory drugs ${ }^{21}$ and this begs the question of whether this increased permeability could allow passage of molecules through the gut wall which could prove to be antigenic in some patients.

In view of the fact that $1 \%$ of the British population have rheumatoid disease, if dietary manipulation therapy were to help only a very small percentage of them it would still be useful. In addition, many patients find it helpful to feel that they are playing some role themselves in the management of their condition, rather than simply being passive recipients of a disease which overwhelms them. In this context their compliance and 
assistance in dietary therapy can prove to be extremely rewarding.

Dietary manipulation has been part of the folklore of rheumatoid management for decades, and there is evidence that many patients regularly manipulate their diet without the knowledge of their doctors. It would seem logical, therefore, particularly in view of the recent uncertainty about the efficacy and/or safety of various drugs used in the management of rheumatoid disease, to determine scientifically whether dietary manipulation has any value in the management of these patients. If not, then surely the old myths should be laid to rest and patients should not be exploited by those who seek to manipulate their diets and to sell literature on the subject. Furthermore, if dietary manipulation has no value, then patients should be left to enjoy their normal food in peace.

If, however, there is a possible role for dietary manipulation in the therapy of rheumatoid patients, then surely it should be examined in the same way that any treatment is examined and its full role should be assessed, whatever that role may be.

\section{References}

1 Catterall W E. Allergy and arthritis. Ann Rheum Dis 1977; 36: 594.

2 Lewin P, Taub S J. Allergic synovitis due to ingestion of English walnuts. JAMA 1936; 106: 2144.

3 Berger $H$. Intermittent hydrarthrosis with an allergic basis. JAMA 1939; 122: 2402-5.

4 Hench P S, Rosenberg E F. Palindromic rheumatism. Proc Mayo Clin 1941; 16: 808-16.

5 Randolph T G. Allergic myalgia. Journal of the Michigan State Medical Society 1951; 50: 487-94.
6 Gudzent F. Testung und Heilbehandlung von Rheumatismus und Gicht mit Spezifischen Allergenen. Deutsche Medes Wochenschr 1935; 61: 901-6.

7 Turnbull $\mathbf{J}$ A. The relation of anaphylactic disturbances to $\overline{\bar{A}}$ arthritis. JAMA 1924; 82: 1757-9.

8 Turnbull $\mathrm{J} \mathrm{A}$. Changes in sensitivity to allergenic foods in arthritis. American Journal of Digestive Diseases 1944; $15: \overline{ }$ 182-90.

9 Zeller M. Rheumatoid arthritis-food allergy as a factor. Ann Allergy 1949; 7: 200-5, 239.

10 Randolph T G. Ecologically oriented rheumatoid arthritis. In: Dickey L D, ed. Clinical ecology. Springfield, Illinois: Thomas, 1976: 201-12.

11 Sköldstam L, Larsson L, Lindström F D. Effects of fasting and lactovegetarian diet on rheumatoid arthritis. Scand J Rheumatolw 1979; 8: 249-55.

12 Hicklin J A, McEwen L M, Morgan J E. The effect of dict in rheumatoid arthritis. Clin Allergy 1980; 10: 463-7.

13 Parke A C, Hughes G R V. Rheumatoid arthritis and food: af case study. Br Med J 1981; 282: $2027-9$.

14 Williams R. Rheumatoid arthritis and food: a case study. $B r$ Med J 1981; 283: 563.

15 Panush R S, Carter R L. Katz P, Kowsari B. Longley S, FinnieS. Diet therapy for rheumatoid arthritis. Arthritis Rheum 1983;윽 26: $462-71$

16 Ziff M. Diet in the treatment of rheumatoid arthritis. Arthritis $\vec{Z}$ Rheum 1983; 26: 457-61.

17 Kroker G P, Stroud R M, Marshall R T, et al. Fasting and rheumatoid arthritis: a multicenter study. In: Dickey L D, ed.马 Clinical ecology. Springfield, Illinois: Thomas, 1984; 2: 137-44.ర

18 Kremer J M, Michalek A V, Lininger L, et al. Effects of manipulation of dietary fatty acids on clinical manifestation $8 \mathrm{f} \overrightarrow{\mathrm{C}}$ rheumatoid arthritis. Lancet 1985; i: $184-7$.

19 Bellamy N, Watson-Buchanan W. Rheumatology seminar rheumatoid arthritis. Hospital Update 1983: 571.

20 Rinkel H J, Lee C H, Brown D W, Willoughby J W, Williams J M. The diagnosis of food allergy. Arch Otolaryngol 1964; 79: 71-9.

21 Bjarnason I, So A, Levi A J, et al. Intestinal permeability and inflammation in rheumatoid arthritis: effects of non-steroidalo anti-inflammatory drugs. Lancet 1984; ii: 1171-3.

\section{Notes}

\section{7th American Orthopaedic Association International Symposium}

The American Orthopaedic Association is sponsoring its 7th International Symposium on 'Frontiers in low back pain', from 17 to 22 August 1986, at the Chicago Marriott Hotel. Further details from William J Kane, $845 \mathrm{~N}$ Michigan Avenue, Chicago, IL 60611, USA.

\section{Conference on drug toxicity and compliance}

The topic for next year's annual day conference in the series 'Growing Points in the Treatment of Rheumatic
Diseases', which will be held on Thursday, 1 May 1986 at the Regional Health Authority, Harrogate, Yorkshire, is̊ 'Drug Toxicity and Compliance'. Further details from Dr Howard Bird, Royal Bath Hospital, Harrogateo HG1 2PS.

\section{Second World Conference on Inflammation}

This conference will be held in Monte Carlo on 19-22。 March 1986 and will discuss the progress made in the fields of antirheumatic drugs, analgesics, antipyretics, and im: munomodulators. Details from the Organizing Secretariat ALM s.r.1., Via Lattuada, 26-20135 Milan, Italy. 